\section{Tratamiento laparoscópico transperitoneal de mielolipoma suprarrenal}

Pineda-Murillo J, ${ }^{1}$ Vergara-Vizuet J, ${ }^{1}$ Hernández-León $0,{ }^{1}$ Torres-Aguilar J, ${ }^{1}$ Viveros-Contreras C, ${ }^{1}$ Marín-Guizar DM ${ }^{2}$

\section{Resumen}

ANTECEDENTES: los tumores suprarrenales lipomatosos son lesiones hormonalmente inactivas, poco frecuentes, con incidencia de $4-5 \%$. El mielolipoma es el tumor adiposo más común de la glándula adrenal. El origen de los mielolipomas suprarrenales es incierto. Los mielolipomas están compuestos por una mezcla inusual de tejido adiposo maduro y elementos hematopoyéticos trilineados, relativamente normales. El objetivo de este estudio es exponer un caso de mielolipoma suprarrenal y su tratamiento médico.

CASO CLÍNICO: paciente femenina de 64 años de edad, con padecimiento de seis meses de evolución, caracterizado por dolor lumbar derecho, acompañado de saciedad temprana y pérdida ponderal de 8 kg. De acuerdo con los hallazgos clínicos se le realizó una urografía por tomografía computada multicorte, que demostró una lesión suprarrenal derecha, sin afectación del polo renal superior ipsilateral, ni reforzamiento con la aplicación del medio de contraste. Se practicó adrenalectomía laparoscópica transperitoneal derecha, que reportó un mielolipoma adrenal.

PALABRAS CLAVE: mielolipoma, suprarrenal, laparoscopia.
Rev Mex Urol. 2017 may;77(3):213-218.

\section{Transperitoneal laparoscopic approach in adrenal myelolipoma}

Pineda-Murillo J, ${ }^{1}$ Vergara-Vizuet J,, ${ }^{1}$ Hernández-León $0,{ }^{1}$ Torres-Aguilar J, ${ }^{1}$ Viveros-Contreras $\mathrm{C}^{1}$ Marín-Guizar DM ${ }^{2}$

\section{Abstract}

INTRODUCTION: Adrenal lipomatous tumors are rare hormonally silent lesions with an incidence of $4-5 \%$. Myelolipoma is the most common fatty tumor of the adrenal gland. Adrenal myelolipoma etiology is still unknown. Myelolipomas are composed of an unusual mixture of mature adipose tissue and relatively normal trilineage hematopoietic elements. The aim of our report was to describe a case of adrenal myelolipoma and its medical approach.

\author{
${ }^{1}$ Departamento de Urología. \\ ${ }^{2}$ Departamento de Patología. \\ Hospital Juárez de México, Ciudad de México. \\ Recibido: diciembre 2016 \\ Aceptado: abril 2017 \\ Correspondencia \\ Javier Pineda Murillo \\ xavpineda@gmail.com
}

Este artículo debe citarse como

Pineda-Murillo J, Vergara-Vizuet J, Hernández-León O, Torres-Aguilar J, Viveros-Contreras C, Marín-Guizar DM. Tratamiento laparoscópico transperitoneal de mielolipoma suprarrenal. Rev Mex Urol. 2017 mayo;77(3):213-218. 
CLINICAL CASE: A 64-year-old woman presented with right lumbar pain of 6-month progression, accompanied with early satiety and an 8-kg weight loss. A multi-detector computed tomography urography scan showed the presence of a single right adrenal lesion with no involvement of the ipsilateral upper kidney pole and no enhancement with the application of contrast medium. Right transperitoneal laparoscopic adrenalectomy was performed, revealing adrenal myelolipoma.

KEY WORDS: Myelolipoma; Adrenal; Laparoscopy

\author{
${ }^{1}$ Departamento de Urología. \\ ${ }^{2}$ Departamento de Patología. \\ Hospital Juárez de México, Ciudad de \\ México. \\ Correspondence \\ Javier Pineda Murillo \\ xavpineda@gmail.com
}

\section{ANTECEDENTES}

Las glándulas suprarrenales son órganos endocrinos complejos compuestos de dos regiones distintas: la corteza y la médula. La corteza se compone de tres zonas: la externa o glomerulosa (sitio de producción de aldosterona), la central o fasciculada (donde se produce cortisol y esteroides sexuales) y la interna o reticular (secreta cortisol, andrógenos y estrógenos). La médula suprarrenal es la región central de la glándula y secreta catecolaminas. ${ }^{1}$ Las glándulas suprarrenales sanas tienen forma de $\mathrm{Y}$ o $\mathrm{V}$ y se localizan en el retroperitoneo, dentro de la fascia de Gerota. La glándula suprarrenal derecha tiende a estar más cefálica que la izquierda. El aporte de sangre arterial a las glándulas se deriva de tres fuentes principales de cada lado y cada glándula suprarrenal recibe ramas de la arteria frénica ipsilateral inferior (arterias suprarrenales superiores), aorta (arterias suprarrenales medianas) y arteria renal (arterias suprarrenales inferiores). El drenaje de la vena suprarrenal derecha desemboca en la vena cava y de la vena suprarrenal izquierda en la vena renal ipsilateral. ${ }^{2}$ Los tumores suprarrenales lipomatosos son lesiones hormonalmente inactivas, poco frecuentes, cuya incidencia varía de $4-5 \%$, a menudo benignas (mielolipomas, lipomas, angiomiolipomas o teratomas maduros) y excepcionalmente malignas (liposarcomas). El mielolipoma es el tumor adiposo más común de la glándula adrenal. Gierke describió, por primera vez, esta enfermedad en 1905 y el término de mielolipoma fue sugerido por Oberling en 1929. Se estima que la prevalencia en autopsias es de $0.08-0.4 \%$. En la actualidad, debido a los avances en las técnicas de de diagnóstico se detectan de manera fortuita en los ultrasonidos o tomografías computadas, por lo que su incidencia es de $2 \%$ de todas las neoplasias suprarrenales. ${ }^{3,4}$

Los tumores suelen aparecer en la edad adulta tardía, entre la quinta y séptima décadas de la vida (límites de edad entre 41-84 años; media de 62), sin predilección por el sexo; la afectación a la glándula adrenal derecha es más común que la izquierda. La localización suele ser intraadrenal, aunque se han descrito sitios extraadrenales (pulmón, mediastino, hígado, estómago, leptomeninges $y$, especialmente, la región presacra). ${ }^{5,6} \mathrm{El}$ origen de los mielolipomas suprarrenales es incierto; sin embargo, se han planteado hipótesis como: a) metaplasia de células corticales suprarrenales o estromales pluripotenciales provocadas por estrés crónico, degeneración y aumento de la concentración de cortisol (estados de hiperactividad), b) hematopoyesis extramedular en situaciones patológicas, c) embolismo de células de la médula ósea y $d$ ) restos embriológicos de la médula ósea. ${ }^{3,7}$ Algunos autores sugieren que el exceso de secreción de hormonas adrenocorticotrópicas durante un 
largo periodo, el estilo de vida estresante contemporáneo y la dieta desequilibrada pueden tener participación en la patogénesis del tumor. El síndrome de Cushing, hiperaldosteronismo, hipertensión, diabetes y obesidad suelen relacionarse con estímulos suprarrenales. Además, se ha informado la coexistencia de mielolipoma en pacientes con hiperplasia adrenal congénita $(\mathrm{CAH})$, debida a deficiencia de 21-hidroxilasa o 17-hidroxilasa y su relación con la translocación cromosómica (3;21) (q25;p11). El mielolipoma también se ha relacionado con enfermedades neoplásicas como: feocromocitoma, carcinoma renal, tumor testicular o de células estromales y antecedentes de cáncer en otras zonas del cuerpo. Hasta el momento se desconoce la relación entre mielolipoma y trastornos del sistema hematopoyético. ${ }^{8-11}$ Los mielolipomas están compuestos por una mezcla inusual de tejido adiposo maduro y elementos hematopoyéticos trilineados, relativamente normales, con sobreproducción de megacariocitos en algunos casos. A diferencia de la médula ósea sana, la celularidad dentro de los mielolipomas varía demasiado y no disminuye conforme avanza la edad del paciente. Estas lesiones no liberan células hematopoyéticas a la circulación, debido a la irregularidad en el soporte del estroma y a la deficiencia de la microvasculatura., ${ }^{2,10,12}$ Los mielolipomas casi siempre son asintomáticos, aunque algunas veces producen dolor por compresión mecánica, debida al tamaño del tumor; pueden o no asociarse con hematuria o disfunción endocrina y hemorragia por ruptura espontánea o necrosis. ${ }^{13,14}$ La resección quirúrgica se recomienda en pacientes con lesiones mayores de 4 o $5 \mathrm{~cm}$, por el riesgo de hemorragia. $^{10}$ En la actualidad, la vía de acceso es laparoscópica. ${ }^{12}$

\section{CASO CLÍNICO}

Paciente de 64 años de edad, sin antecedentes heredofamiliares de importancia para el padeci- miento actual. Entre sus antecedentes personales patológicos reportó hipertensión arterial sistémica y diabetes mellitus tipo 2, ambas de larga evolución, en tratamiento irregular con telmisartán e insulina intermedia. Acudió a consulta por un padecimiento de seis meses de evolución, caracterizado por dolor lumbar derecho, punzante, de intensidad moderada, sin irradiaciones, con periodos de remisión y exacerbación, acompañado de saciedad temprana y pérdida ponderal de $8 \mathrm{~kg}$ en ese periodo. A la exploración física se observó constitución mesomórfica, con escala del estado de funcionamiento de $90 \%$ (Karnofsky Performance Scale Index), signos vitales dentro de los parámetros normales, sistema cardiopulmonar sin alteraciones, abdomen globoso, con peristalsis normal, con tumoración palpable en el hipocondrio derecho y epigastrio, de $5 \times 6$ $\mathrm{cm}$, aproximadamente, blanda y móvil, signo de Giordano bilateral negativo, sin datos de irritación peritoneal. Los genitales y extremidades se observaron sin alteraciones.

De acuerdo con los hallazgos clínicos se realizó una urografía por tomografía computada multicorte (UROTAC), que demostró una lesión suprarrenal derecha, de $8.1 \times 6.1 \times 9.0 \mathrm{~cm}$, con bordes regulares y definidos, hipodensa, septada, índice de atenuación de -93 unidades Hounsfield, sin afectación del polo renal superior ipsilateral, ni reforzamiento con el medio de contraste (Figura 1). Los estudios de laboratorio complementarios mostraron concentraciones séricas de cortisol, metanefrinas en plasma y orina, y ácido vanililmandélico sin alteraciones, con lo que se estableció la disfunción hormonal del tumor. Debido a la intensidad del dolor y al tamaño tumoral se realizó adrenalectomía laparoscópica transperitoneal derecha por medio de tres puertos de acceso, cuyo hallazgo histopatológico reportó un espécimen de 20 g, de 12 x $11 \times 12 \mathrm{~cm}$, con superficie lisa, blancoamarilla y anfractuosa naranja, con fragmentos fibroadiposos y células hematopoyéticas, sin 


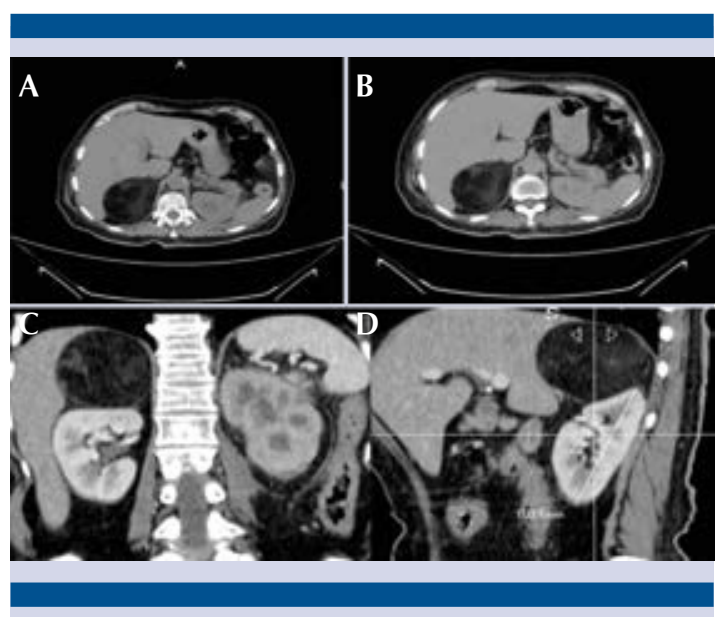

Figura 1. Urotomografía en corte axial simple (A), contraste (B), coronal (C) y sagital (D): lesión suprarrenal derecha, de $8.1 \times 6.1 \times 9.0 \mathrm{~cm}$, con índice de atenuación de -93 unidades Hounsfield, sin reforzamiento al medio de contraste.

evidencia histológica de malignidad, compatible con mielolipoma adrenal (Figura 2). No se observaron complicaciones posoperatorias; la paciente evolucionó favorablemente y egresó al tercer día de hospitalización. Actualmente continúa en seguimiento por los servicios de Urología y Endocrinología, encontrándose asintomática.

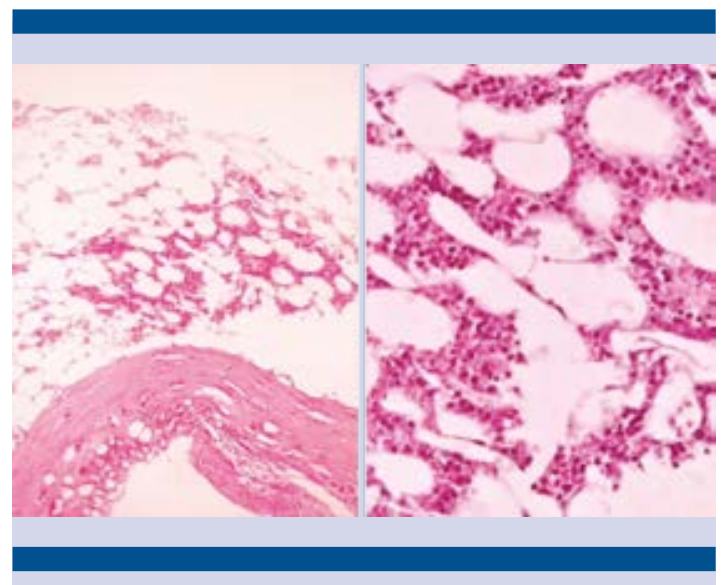

Figura 2. Mezcla variable de grasa madura, con nidos de precursores granulocíticos, eritroblastos y megacariocitos (tinción con hematoxilina-eosina [H-E]).

\section{DISCUSIÓN}

Para establecer el diagnóstico de alguna neoplasia suprarrenal se requieren estudios bioquímicos y hormonales (determinación sérica de cortisol, metanefrinas, ácido vanililmandélico), ya sea mediante la obtención de muestras de sangre u orina, con la finalidad de descartar la funcionalidad del tumor. ${ }^{3,15,16}$ La tomografía axial computada (TAC) es el estudio de elección para detectar las masas suprarrenales (técnica con mayor sensibilidad para detectar la densidad grasa $[-30$ a $-100 \cup H]$ ). Generalmente muestra un tumor bien circunscrito en su cápsula, con o sin calcificaciones en $20 \%$ de los tumores, ya sean irregulares o difusas. Al aplicar el medio de contraste se ve reforzada y puede observarse una lesión heterogénea, con zonas hipodensas de diferente atenuación cuando se asocia con hemorragia reciente o antigua. El tamaño tumoral representa un factor de riesgo para lesiones funcionales y malignas, sobre todo en mayores de $4 \mathrm{~cm}$ (sensibilidad de $93 \%$ y especificidad de $42 \%$ ). La resonancia magnética nuclear es un estudio de imagen adicional para establecer el diagnóstico de adenomas con elevada cantidad de lípidos y para diferenciar los que tienen deficiencia de lípidos de otras lesiones más agresivas. Proporciona una señal hiperintensa para definir la grasa en un mielolipoma suprarrenal en las secuencias T1 y T2; el tejido hematopoyético tiene una proyección hipointensa en $\mathrm{T} 1$, con intensidad moderada en T2, y la falta de uniformidad en la textura de la grasa y los elementos hematopoyéticos proporciona una apariencia heterogénea en la secuencia T2. La hemorragia en la glándula suprarrenal muestra una imagen hiperintensa en T1, que al aplicar el medio de contraste no se refuerza; por tanto, se distingue, principalmente, de feocromocitomas o tumores malignos que se refuerzan intensamente al aplicarlo. ${ }^{17,18}$ La PET/CT está diseñada para evaluar la actividad metabólica del tejido. El radiotrazador más utilizado es la 18-fluorodeoxiglucosa (18F- 
FDG), que ha demostrado un alto rendimiento diagnóstico en la diferenciación de las masas suprarrenales benignas y malignas, con altos índices de sensibilidad y especificidad, superiores a 95 y 90\%, respectivamente. En pacientes con mielolipomas los elementos hematopoyéticos pueden absorber la 18-FDG. La sensibilidad para lesiones menores de $1 \mathrm{~cm}$ es baja. ${ }^{17,18} \mathrm{La}$ biopsia por aspiración con aguja fina, descrita por Deblois y Demay, es un recurso diagnóstico. El diagnóstico diferencial se establece con: lipoma retroperitoneal, liposarcoma retroperitoneal, angiomiolipoma renal exofítico, neoplasia suprarrenal primaria o metastásica. Aunque el tratamiento suele ser conservador, algunos estudios sugieren la intervención quirúrgica en pacientes con mielolipomas sintomáticos, en crecimiento o mayores de $6 \mathrm{~cm}$ para reducir el riesgo de dolor abdominal o ruptura y hemorragia potencialmente mortal. ${ }^{5}$

Gagner realizó la primera adrenalectomía laparoscópica, en 1992, por vía transperitoneal para tener acceso a la glándula suprarrenal. En la actualidad, el acceso laparoscópico constituye una de las principales indicaciones de cirugía urológica, dejando atrás realizar amplias incisiones lumbotómicas para tratar lesiones suprarrenales, que anteriormente provocaban elevada morbilidad y mortalidad, incluso de $40 \%$. Debe considerarse la técnica laparoscópica como patrón de referencia en el tratamiento de lesiones benignas funcionales o no de la glándula suprarrenal, es decir, menores de $12 \mathrm{~cm}$. La adrenalectomía laparoscópica transperitoneal puede realizarse mediante acceso anterior supino o lateral. El primero permite efectuar adrenalectomía bilateral sin reposicionar al paciente y el segundo dispone de mayor espacio de trabajo. El paciente se coloca en posición inclinada, hacia atrás, de 30 a 45 grados. Después de insuflar el abdomen se colocan tres o cuatro trócares a lo largo del margen costal derecho. El duodeno se diseca mediante la maniobra de Kocher y se utiliza un trocar para la retracción del hígado. La vena renal y la suprarrenal se disecan. Pueden utilizarse clips quirúrgicos: dos en el lado de la vena y uno en el lado de la pieza. El bisturí armónico es efectivo para realizar la disección. La muestra se coloca en un saco endoscópico y se extrae. ${ }^{2,19,20}$ Desde el punto de vista histopatológico, el mielolipoma suprarrenal se compone de tejido adiposo y elementos hematopoyéticos rodeado por una pseudocápsula. Desde la perspectiva macroscópica, el color varía de rojo intenso a amarillo pálido, debido a la gran cantidad de precursores hematopoyéticos y grasa. La superficie cortada puede revelar áreas focales de infarto o hemorragia. En la vista al microscopio, se compone de una mezcla variable de grasa madura, con islas y nidos de precursores granulocíticos inmaduros y maduros, eritroblastos y megacariocitos. En algunos casos se observan áreas de infarto, hemorragia y, rara vez, focos de osificación.

Soos clasificó los mielolipomas en dos categorías: Tipo I, formaciones tumorales de color amarillo-naranja y predominio de tejido conectivo graso y Tipo II, formaciones tumorales de color rojo oscuro-pardo, con predominio de eritroblastos. La tinción inmunohistoquímica y las pruebas moleculares no tienen ninguna ventaja clínica o histológica. 7,8,21,22

\section{CONCLUSIÓN}

Los mielolipomas son tumores poco frecuentes de las glándulas suprarrenales, que casi siempre se detectande manera fortuita en los estudios de imagen. Las indicaciones para su tratamiento quirúrgico son similares a las de los incidentalomas. La suprarrenalectomía por vía laparoscópica es una técnica quirúrgica segura, con bajo porcentaje de complicaciones y necesidad de menor estancia hospitalaria. Es importante reconocer los criterios diagnósticos y de tratamiento médico o quirúrgico para ofrecer a los pacientes las mejores alternativas. 


\section{Financiamiento}

No se recibiố patrocinio de ningún tipo para Ilevar a cabo este estudio.

\section{Conflicto de intereses}

Los autores declaran no tener conflicto de intereses.

\section{REFERENCIAS}

1. Brian CA, Isaac RF. Adrenal Imaging and Intervention. Radiol Clin N Am 2015;(53):1021-1035.

2. Wein JA, Kavoussi RL, Partin WA, et al. Campbell-Walsh Urología. 11a Ed. China: Elsevier; 2016.

3. Martínez-Cornelio A, Hernández-Toriz N, Alvarado-Cabrero I, et al. Mielolipoma suprarrenal, revisión de casos en México. Rev Mex Urol 2008;68(6):334-340.

4. Sz-Wen $\mathrm{H}$, Kenneth $\mathrm{S}$, Wei-Ching $\mathrm{L}$, et al. Adrenal myelolipoma: A 10-year single-center experience and literature review. Kaohsiung J Med Sci 2012;(28):377-382.

5. Ramírez M, Misra S. Adrenal myelolipoma: To operate or not? A case report and review of the literature. Int J Surg Case Rep 2014;(5):494-496.

6. Olivar-Roldán J, Molina-Baena B, Pavón De Paz I, et al. Mielolipoma adrenal gigante: revisión a propósito de un caso. Endocrinol Nutr 2008;55(3):139-41.

7. Olobatuyi FA, MacLennan GT. Myelolipoma. J Urol 2006;(176):1188.

8. Khater N, Khauli R. Myelolipomas and other fatty tumours of the adrenals. Arab J Urol 2011;(9):259-265.

9. Jia-Hwia W. Detection and Treatment of Adrenal Myelolipomas. Urol Sci 2011;22(1):38-39.
10. Zuviri-González A, Espinosa-Pérez G, Guzmán-Hernández $\mathrm{F}$, et al. Mielolipoma supradrenal. Rev Mex Urol 2011;71(3):188-191.

11. Daneshmand S, Quek ML. Adrenal Myelolipoma: Diagnosis and Management. J Urol 2006;3(2):71-74.

12. Cantellano-Orozco M, Merayo-Chalico C, Shuck-Bello C, et al. Patología quirúrgica de la glándula suprarrenal en 20 años: casos del Hospital General Dr. Manuel Gea González. Rev Mex Urol 2009;69(5):238-242.

13. Kumar S, Jayant K, Prasad S, et al. Rare adrenal gland emergencies: A case series of giant myelolipoma presenting with massive hemorrhage and abscess. Nephro Urol Mon 2015;7(1):e22671.

14. Da-Rae K, Yoon Young J, Myung-Won Y, et al. Adrenal Myelolipoma Presenting with Spontaneous Rupture and Retroperitoneal Hemorrhage: A Case Report. J Korean Soc Radiol 2016;75(4):296-299.

15. Young WF. The Incidentally Discovered Adrenal Mass. N Engl J Med 2007;(356):601-610.

16. Zeiger MA, Thompson GB, Duh QY. AACE/AAES Adrenal Incidentaloma Guidelines. Endocr Pract 2009;15(1):1-20.

17. Baltzer $P$, Clauser $P$, Klatte $T$, et al. Work-up of the Incidental Adrenal Mass. Eur Urol Focus 2016;(1):217-222.

18. Fassnacht M, Arlt W, Bancos I, et al. Management of adrenal incidentalomas: European Society of Endocrinology Clinical Practice Guideline in collaboration with the European Network for the Study of Adrenal Tumors. Eur J Endocrinol 2016;(175):1-34.

19. Virseda JA, Ruiz-Mondéjar R, Donate MJ, et al. Suprarrenalectomía laparoscópica transperitoneal. Actas Urol Esp 2011;35(9):546-551.

20. Yamashita S, Ito K, Furushima K, et al. Laparoscopic versus open adrenalectomy for adrenal myelolipoma. Ann Med Surg 2014;(3):34-38.

21. Todorov G, Lukanova T, Georgiev M. Surgical treatment of adrenal myelolipoma. J Clin Med 2010;3(3):24-30.

22. Kumar V, Abbas A, Fausto N. Robbins y Cotran, Patología estructural y funcional. 7ạ Ed. España: Elsevier; 2008. 